\title{
Políticas para el Desarrollo de Sistemas de Innovación en México
}

\author{
Innovation System Development Policies in Mexico
}

\author{
José Luis Solleiro', Claudia Gaona², Rosario Castañón ${ }^{3}$
}

\begin{abstract}
In 20II, the Mexico-State Council of Science and Technology in alliance with the Inter-American Development Bank and the National Council of Science and Technology implemented a program to promote collaborative networks among firms for improving competitiveness trough innovations. Target firms belong to priority manufacturing and services sectors. This paper deals with the analysis of the program, State of Mexico's innovation capacities are described, the institutional framework and the main actors are also analyzed.

The results show that SMEs had lack of experience in innovation but they increased their investment in innovation projects. Based on that analysis we conclude that success of innovation policies is highly dependent on previous experience and learning abilities of firms. Finally, we draw some recommendations for building capacities at the firm level as well as for strengthening innovation policies aimed at involving and technically upgrading SMEs
\end{abstract}

Keywords: Mexico-State Council of Science and Technology; Inter-American Development Bank; innovation policies; SMEs

En 20II, el Banco Interamericano de Desarrollo, el Consejo Mexiquense de Ciencia y Tecnología y el Consejo Nacional de Ciencia y Tecnología financiaron un programa para impulsar redes de colaboración tecnológica en sectores económicos prioritarios del Estado de México e integrar un sistema local de innovación. En este trabajo se analiza dicho programa, se describe la capacidad de innovación del Estado, su marco institucional y los principales actores. Los resultados obtenidos fueron sumamente positivos, en particular respecto a la inversión de pequeñas y medianas empresas en proyectos tecnológicos. Este programa puede ser un modelo para el desarrollo de políticas de innovación en el país al identificar elementos clave de su éxito, como por ejemplo: la experiencia previa de las empresas en materia de innovación y sus capacidades de aprendizaje.

Keywords: Consejo Mexiquense de Ciencia y Tecnología; Banco Interamericano de Desarrollo; políticas de innovación; PyMES

\footnotetext{
1,3 Centro de Ciencias Aplicadas y Desarrollo Tecnológico, Universidad Nacional Autónoma de México. e-mail: 'solleiro@unam.mx, ${ }^{3}$ rosarioc@unam.mx

${ }^{2}$ Consejo Nacional de Ciencia y Tecnología.
}

ISSN: 07 I8-2724. (http://www.jotmi.org) 


\section{Introducción}

El Estado de México (EdoMex) está localizado en la región central de México y por su población y contribución al Producto Interno Bruto (PIB) es uno de los estados más importantes del país ${ }^{1,2}$.

Más del $99 \%$ de las unidades económicas localizadas en el EdoMex pertenecen a la categoría de micro, pequeñas y medianas empresas; las grandes compañías representan sólo el $0.2 \%$ del total. Respecto al empleo, las microempresas generan el $48.2 \%$, las pequeñas y medianas el $28.8 \%$ y las grandes aproximadamente el $5 \%$.

A pesar de su importancia económica, el Estado de México se encuentra abajo del promedio en los diferentes indicadores de competitividad. De acuerdo al análisis del Instituto Mexicano para la Competitividad (IMCO, 20 I0), en 2010 el Estado ocupó el lugar número 28, de 32 posibles.

El Informe de Innovación Regional, elaborado por la OCDE (2009), indica que la mayor proporción del valor agregado bruto (VAB) del Estado proviene de los sectores de media y baja tecnología. Las industrias de baja tecnología representaron el $41.9 \%$ del VAB, las de media tecnología el 23.3 y las de alta tecnología el 8.8\%.Así, una de las prioridades estratégicas para contender con la falta de competitividad es fortalecer la innovación incrementando las inversiones del sector privado en actividades de investigación y desarrollo (lyD) y mejorar la cooperación entre empresas, instituciones de investigación, universidades y el gobierno.

Investigaciones previas han mostrado que el apoyo a las pequeñas y medianas empresas (PYMEs) en lo referente al fortalecimiento de sus capacidades de innovación, deriva en la mejora de su competitividad (Dini et al., 2007; ECLAC, 2010; ECLAC - IDB - OAS, 20II).

México ha realizado cambios drásticos en su política económica durante los últimos 30 años, priorizando la apertura comercial. Aunque las exportaciones han crecido, la competitividad de México ha caído en la última década. Los indicadores de innovación del país son cada vez peores debido a los pocos recursos destinados a actividades de lyD, así como a la débil articulación entre los actores del sistema de innovación.

A principios de la década, autoridades locales reconocieron la necesidad de establecer nuevas iniciativas de innovación enfocadas a la solución de los retos sociales, lo que condujo al concepto de sociedades y redes de innovación. Este concepto debería de materializar varias de las características ideales de una agenda efectiva de lyD, tales como coherencia, un enfoque de lyD integrado, innovación y educación, el establecimiento de condiciones favorables, la promoción de la cooperación entre actores, buena gobernanza, y presupuestos adecuados.

Ante esta situación, se establecieron algunas iniciativas con carácter regional para incrementar las inversiones y participación de actores en la innovación. Concretamente se crearon los consejos estatales de ciencia y tecnología y fondos de apoyo a la lyD para sectores específicos.

En este contexto, el Consejo Mexiquense de Ciencia y Tecnología (COMECYT), en colaboración con el Banco Interamericano de Desarrollo (BID) y el Consejo Nacional de Ciencia y Tecnología (CONACYT), implementó el programa denominado Sistema de Innovación del Estado de México (SIEM), cuyo objetivo central fue mejorar la competitividad de las PYMES del EdoMex mediante:

i. Incentivos y apoyo técnico para las PYMEs con alto potencial de innovación

ii. Promoción de redes empresariales para concretar innovaciones

iii. Fortalecimiento de vínculos entre universidades y el sector privado en nueve sectores industriales identificados como prioritarios (alimentos procesados, floricultura, química, industria farmacéutica, industria textil y del vestido, plástico, logística, manufactura avanzada y tecnologías de la información). ${ }^{3}$

Estas actividades fueron apoyadas mediante talleres dirigidos a funcionarios de gobierno, industriales e investigadores $y$ orientados a la capacitación en temas básicos de gestión tecnológica así como en la definición y evaluación de políticas de innovación.

El punto de partida del Programa SIEM fue definir la agenda estatal de innovación a partir del diagnóstico de las capacidades y recursos para innovar, así como de la existencia de instrumentos de política y la conducción de procesos para establecer prioridades en donde participaron representantes de empresas, instituciones de investigación y el gobierno estatal. En función de los resultados de la agenda estatal, se promovieron redes sectoriales para facilitar la interacción y colaboración entre empresas e instituciones de investigación.

\footnotetext{
' El EdoMex tiene aproximadamente 15 millones de habitantes y es el segundo Estado más densamente poblado, solo detrás de la Ciudad de México. ${ }^{2}$ El PIB del Estado de México es de $\mathbf{9 2 . 8}$ mil millones de dólares, lo que representa el $9.4 \%$ de la economía nacional, ocupando el segundo lugar en importancia en lo que se refiere a este rubro.

${ }^{3}$ Los nueve sectores industriales estratégicos para el Estado de México fueron seleccionados mediante estudios previos elaborados por la Secretaría de Desarrollo Económico del Estado de México.
} 
A las PYMEs del Estado de México se les ofrecieron fondos concurrentes para proyectos de innovación tecnológica como un incentivo económico para promover la inversión privada y los vínculos institucionales.

El programa SIEM fue un proyecto piloto del cual se generó experiencia para el diseño de un modelo que pueda ser reproducido en otros Estados del país.

En este trabajo se analiza la experiencia del programa SIEM, se identifican factores de éxito y los obstáculos para la definición de una agenda de innovación, así como los principales problemas para la implementación de políticas gubernamentales relacionadas con el Programa SIEM. Con base en este análisis, y a la participación que los autores tuvieron como coordinadores y promotores de las redes de innovación dentro del SIEM, se elaboran un conjunto de recomendaciones para mejorar la efectividad en la promoción de sistemas estatales de innovación orientados a PYMEs.

\section{Metodología}

El enfoque seguido en el análisis del Programa SIEM es el de "investigación - acción", en el cual se promueve el cambio y al mismo tiempo se extraen lecciones aprendidas durante el proceso. Los autores de este documento estuvieron involucrados en diferentes etapas del programa SIEM como coordinadores de todo el proceso y como agentes facilitadores para la construcción de algunas agendas sectoriales de innovación, la identificación de proyectos de innovación y la construcción de redes de colaboración. Esta participación facilitó el acceso a información clave sobre el proceso y los actores involucrados.

El concepto de investigación - acción fue propuesto por Kurt Lewin (1946) como una forma de investigación que permite la articulación de un enfoque experimental de las ciencias sociales con las actividades de acción social para responder a los problemas actuales. Mediante la investigación - acción se pretende abordar de manera simultánea la generación de conocimiento y el cambio social con la idea de combinar la teoría y la práctica.

La esencia de la investigación - acción es que los investigadores sean participantes y el proceso de cambio es en sí mismo el sujeto de la investigación. En este proceso los roles pueden intercambiarse (Checkland, 1999) y es muy importante reducir la generación de consensos alrededor de los hallazgos de la investigación.
Lewin (1958) considera que la investigación - acción es altamente participativa y está asociada a un proceso de tres etapas. La primera está caracterizada por el trabajo de un grupo dedicado a identificar la necesidad del cambio. En la segunda etapa, se hace el diagnóstico del problema y se proponen y prueban nuevos enfoques. Normalmente, se plantean hipótesis y la acción permite la validación de éstas. En la tercera etapa se evalúa el cambio y los efectos de la acción.

Como puede observarse, la implementación del programa SIEM tuvo un componente de participación muy fuerte que involucró a los diferentes agentes a los que se orientó el programa y un papel muy activo por parte de los autores de este trabajo en las diferentes etapas del SIEM.

\section{Resultados y Discusión}

\section{I Sistemas regionales de innovación y la agenda de innovación}

Actualmente hay un acuerdo generalizado entre la academia, respecto a que conocimiento, aprendizaje e innovación son los factores más importantes para la competitividad en un mundo globalizado y en economías basadas en conocimiento (Lundvall, 1992). En este sentido, el tema de innovación ocupa los primeros lugares dentro de las políticas industrial y regional (Tödtling and Trippl, 2005).

La política de innovación basada en el modelo lineal fue predominante hasta principios de los 90's. En este contexto, la política industrial convencional orientada a la manufactura no consideró las deficiencias organizacionales y gerenciales de las empresas, en particular de las pequeñas y medianas (Lagendijk, 2000). Los instrumentos fueron orientados, en la mayoría de los casos, hacia las empresas como entes individuales y aplicados de forma descoordinada (Asheim et al., 2003). El concepto de sistema regional de innovación (SRI) ha ganado peso, desde el inicio de los 90's, entre quienes diseñan las políticas y el sector académico toda vez que el concepto se basa en un modelo de innovación interactivo.

La característica clave de este concepto se refiere a que la capacidad de una economía para generar innovaciones se basa en la forma en que interactúan los actores del sistema, es decir, las empresas, las universidades, centros de investigación, instituciones gubernamentales, etc. La innovación se concibe como un proceso social que se desarrolla más exitosamente con la construcción de redes, en donde existe una fuerte interacción entre proveedores y compradores de bienes, servicios, conocimiento y tecnología, incluyendo la infraestructura de organizaciones públicas que generan conocimiento, tales como 
universidades y centros públicos de investigación (Hae, 2006). Este enfoque ha sido bien aceptado como un marco de referencia adecuado para avanzar en la comprensión del proceso de innovación en una economía regional (Asheim et al., 2003; Isaksen, 200 I; Cooke, 2002).

La literatura y algunos casos de estudio demuestran que no existe el "modelo ideal" para una política de innovación. Investigaciones empíricas demuestran que las precondiciones para la innovación así como para la conformación de redes difiere fuertemente entre regiones centrales y periféricas. El enfoque de SRI permite considerar estas diferencias al considerar las fortalezas y debilidades de varios subsistemas, clusters $y$ las interdependencias entre las regiones $y$ sus alrededores.

De acuerdo con experiencias internacionales, en la conformación de agendas de innovación, el primer paso es poner en contacto a todos los grupos de interés (stakeholders) relacionados con el proceso de innovación con la finalidad de elaborar una visión de largo plazo (CORDIS, 2004).

El paso siguiente, en la elaboración de la agenda, es establecer prioridades de lyD para el mediano y largo plazo, para lo cual es indispensable obtener las contribuciones de todos los grupos de interés que deriven en una lista de programas, incluyendo medidas para mejorar las capacidades científicas y tecnológicas de la región. Para ello se requiere considerar el marco de referencia tecnológico (incluyendo políticas sectoriales y temas regulatorios, derechos de propiedad intelectual, etc.) y el medio ambiente de negocios para la futura introducción al mercado.

Para que las prioridades se traduzcan en programas factibles es necesario diseñar mecanismos que promuevan las inversiones públicas y privadas, por ello es crítico involucrar a la comunidad financiera así como a los fondos gubernamentales.

Para asegurar la futura implementación de las tecnologías de medio y largo plazo se deben identificar los retos y acciones que garanticen oportunidades de educación y entrenamiento para contar con personal calificado en las áreas de políticas públicas e investigadores.

También, es indispensable establecer un proceso continuo de comunicación que mejore el diálogo entre los distintos actores ( industria, gobierno y academia) para lograr los objetivos de la agenda.
Los principales actores relacionados con la construcción de la agenda son (CORDIS, 2004):

- Industria: empresas pequeñas, medianas y grandes, abarcando toda la cadena de producción y suministro, incluyendo los subsistemas de proveedores de componentes, equipo e industrias usuarias.

- Investigadores involucrados en la transferencia de tecnología y el desarrollo comercial de tecnologías (por ejemplo proveedores de servicios y operadores). - Autoridades públicas, en su papel de hacedores de política y agencias de financiamiento, así como promotores y consumidores de tecnologías.

- Instituciones de investigación y la comunidad académica, fortaleciendo especialmente la interface industria/academia.

- Comunidad financiera: banca privada, fondos de inversión, capital de riesgo, incubadoras de empresas, etc.

- Sociedad civil, incluyendo usuarios y consumidores, asegurando que las agendas de innovación propicien un diálogo abierto entre la comunidad cientíica y la sociedad y que ello sea la base de futuros clientes.

\subsection{Definición de la agenda de innovación del Estado de México}

Se organizaron talleres de consenso para cada uno de los sectores establecidos como prioritarios. Representantes de la industria, la academia y el gobierno participaron bajo la coordinación de un consultor que facilitó el diálogo entre los diferentes actores ${ }^{4}$.

Se elaboró una metodología común para los nueve sectores, la cual contempló los siguientes aspectos:

Actividades previas a los talleres:

- Diagnóstico general del desempeño económico de cada sector dentro del Estado de México

- Identificación de PYMEs con potencial para realizar actividades de innovación. ${ }^{5}$

\footnotetext{
${ }^{4}$ El desempeño de los consultores fue muy heterogéneo lo que derivó en dificultades para la conducción adecuada de los talleres así como en la definición de las agendas de innovación sectoriales. Especialistas con conocimiento de los sectores y gestión tecnológica, así como con habilidades para coordinar los talleres de generación de consensos son escasos, lo que sin duda dificultó la tarea de elaborar las agendas.

${ }^{5}$ Se utilizaron fuentes de información públicas, tales como el Registro Nacional de Instituciones y Empresas Científicas y Tecnológicas, directorios especializados de los sectores, directorios de las empresas beneficiadas con fondos públicos para el desarrollo de tecnología, estadísticas de patentes, asociaciones profesionales y referencias de empresas obtenidas a través de cámaras industriales.
} 
- Análisis de las principales tendencias científicas y tecnológicas que permitieran tener una visión de los posibles escenarios, oportunidades y amenazas.

Actividades realizadas durante los talleres:

- Análisis de la situación actual del sector industrial, la existencia de capacidades científicas y tecnológicas y las tendencias futuras.

- Identificación de las capacidades de instituciones locales para desarrollar y transferir tecnologías, así como proveer servicios técnicos útiles para innovar. - Análisis de posibles proyectos que pudieran realizarse por un grupo de actores del sistema de innovación.

- Evaluación de los proyectos potenciales para definir la lista de prioridades. Los criterios de selección fueron: disponibilidad de empresas interesadas, impacto potencial sobre necesidades sociales, oportunidad de mercado (tamaño y dinámica); capacidades para desarrollar e implementar el proyecto, y disponibilidad de recursos.

- Agenda preliminar, incluyendo la lista de prioridades, ejemplos de las redes que se deberían construir para ejecutarlos y recomendaciones de política para apoyar programas y proyectos.

Actividades posteriores a los talleres:

- Difusión de las agendas de cada sector

- Presentación de la agenda a diferentes grupos de interés

- Formulación de proyectos para conseguir fondos para su ejecución

Los talleres fueron complejos y los problemas y dificultades encontrados tuvieron su origen en las diferentes expectativas que tenían los participantes, los distintos niveles de información sobre la problemática del sector y conocimiento sobre las tendencias científicas y tecnológicas.

En la tabla I, se presentan los principales aspectos que impactaron el proceso de generación de la agenda. Es claro que un buen enfoque metodológico ${ }^{6}$, una buena organización y la disponibilidad de fuentes de información de valor agregado fueron factores positivos.
Los factores negativos de mayor relevancia fueron la burocracia de los órganos gubernamentales y la falta de conocimiento técnico. Es importante señalar que el personal técnico de las empresas, en general, no estaba familiarizado con el concepto de innovación y las principales tendencias tecnológicas de su sector.

El coordinador de los talleres jugó un papel importante. En los tres casos en los que los consultores no cumplieron con las expectativas, los resultados entregados no fueron útiles para establecer la agenda y generó descontento entre los participantes. Por el contrario, los coordinadores más activos motivaron a los empresarios a que participaron en los talleres y generaron alto consenso respecto a las prioridades.

Durante la ejecución de los talleres, se presentaron algunos aspectos que hicieron difícil llegar a conclusiones, entre éstos destacan los siguientes:

- Falta de conocimiento, de algunos participantes, sobre el contexto, principales indicadores y tendencias del sector. Esta circunstancia hizo evidente que la selección de los participantes no debe basarse en cuestiones políticas.

- Falta de comprensión sobre los procesos de innovación tecnológica y organizacional y su papel estratégico en la competitividad de las empresas. Esta situación mostró la necesidad de un cambio cultural profundo.

- Las brechas respecto al nivel de información de los empresarios en tópicos básicos relacionados con la innovación, comportamiento del mercado y la competencia, dificultaron la identificación de los problemas tecnológicos principales en los que puede tener injerencia el Estado.

- Los representantes de la industria se mostraban recelosos de compartir información sobre sus problemas $\mathrm{u}$ oportunidades tecnológicas argumentando que era información confidencial. Ante esta circunstancia, en algunos casos fue posible hacer visitas individuales y conseguir información, pero no se logró la interacción e intercambio de ideas.

A pesar de los obstáculos y limitaciones, el trabajo de los consultores permitió generar elementos suficientes para estructurar la agenda de innovación.

\footnotetext{
${ }^{6} \mathrm{~A}$ pesar de que los consultores tenían una guía metodológica, la implementación de ésta se realizó de diversas maneras, lo que también mostró resultados diferentes.
}

ISSN: 07 I8-2724. (http://www.jotmi.org) 


\begin{tabular}{|c|c|c|c|}
\hline Etapa & Guías de Acción & Obstáculos & Observaciones \\
\hline Pre-taller & $\begin{array}{l}\text { Definición de una metodología } \\
\text { común } \\
\text { Disponibilidad de recursos y organi- } \\
\text { zación } \\
\text { Análisis de la situación económica y } \\
\text { tendencias científicas, } \\
\text { Consejo asesor de alto nivel }\end{array}$ & $\begin{array}{l}\text { Falta de especialistas en los } \\
\text { sectores analizados } \\
\text { Procedimientos burocráti- } \\
\text { cos para la contratación de } \\
\text { consultores } \\
\text { Escasa información sobre los } \\
\text { sectores industriales } \\
\text { Poca confianza en las insti- } \\
\text { tuciones gubernamentales }\end{array}$ & $\begin{array}{l}\text { Los representantes } \\
\text { del sector industrial se } \\
\text { mostraron desconfiados } \\
\text { debido a experiencias } \\
\text { negativas previas. } \\
\text { Retrasos en las actividades } \\
\text { debido a procedimientos } \\
\text { burocráticos muy largos }\end{array}$ \\
\hline $\begin{array}{l}\text { Ejecución de los } \\
\text { talleres }\end{array}$ & $\begin{array}{l}\text { Buena estructura organizacional } \\
\text { Disponibilidad de recintos industri- } \\
\text { ales } \\
\text { Participación de líderes de asocia- } \\
\text { ciones industriales } \\
\text { Coordinación y conocimiento de } \\
\text { algunos consultores }\end{array}$ & $\begin{array}{l}\text { Falta de experiencia y lider- } \\
\text { azgo de tres consultores. } \\
\text { El nivel de información entre } \\
\text { los participantes fue het- } \\
\text { erogénea } \\
\text { Dificultad para identificar } \\
\text { escenarios futuros } \\
\text { Falta de continuidad en los } \\
\text { grupos de trabajo } \\
\text { Falta de conocimiento } \\
\text { técnico } \\
\text { Agendas ocultas }\end{array}$ & $\begin{array}{l}\text { Los consultores que } \\
\text { fallaron en la coordi- } \\
\text { nación de sus actividades } \\
\text { provocaron pérdida de } \\
\text { motivación y carencia de } \\
\text { resultados relevantes. } \\
\text { Algunas actividades tu- } \\
\text { vieron que repetirse y esto } \\
\text { retrasó el programa de } \\
\text { actividades. }\end{array}$ \\
\hline Post-talleres & $\begin{array}{l}\text { Interés de las empresas por recibir } \\
\text { apoyo financiero para sus proyectos } \\
\text { Momentum político con el nuevo } \\
\text { Gobernador } \\
\text { Algunas instituciones académicas } \\
\text { estuvieron dispuestas a participar en } \\
\text { redes de colaboración } \\
\text { Los consultores proporcionaron } \\
\text { asistencia técnica para formular los } \\
\text { proyectos específicos } \\
\text { Capacitación en gestión tecnológica }\end{array}$ & $\begin{array}{l}\text { Procedimientos burocráticos } \\
\text { para disponer de los apoyos } \\
\text { económicos } \\
\text { Recursos escasos para apoy- } \\
\text { ar los proyectos } \\
\text { Falta de experiencia para } \\
\text { desarrollar políticas de } \\
\text { innovación. } \\
\text { Desconfianza de colaborar } \\
\text { con otras empresas } \\
\text { Falta de compromiso de las } \\
\text { empresas para invertir en } \\
\text { proyectos prioritarios } \\
\text { Falta de procedimientos } \\
\text { institucionales para la } \\
\text { transferencia de tecnología y } \\
\text { construcción de redes } \\
\text { Falta de difusión de la agen- } \\
\text { da y retraso en su entrega a } \\
\text { funcionarios de alto nivel }\end{array}$ & $\begin{array}{l}\text { Los retrasos en la dis- } \\
\text { ponibilidad de los recursos } \\
\text { económicos desmotivó a } \\
\text { muchos industriales } \\
\text { El proceso para acceder a } \\
\text { recursos para proyectos } \\
\text { tecnológicos fue complejo } \\
\text { para las PYMEs } \\
\text { Capacitación y asisten- } \\
\text { cia técnica es altamente } \\
\text { apreciada } \\
\text { Muchas universidades } \\
\text { no tienen mecanismos } \\
\text { institucionales, experien- } \\
\text { cia y capacidades para } \\
\text { responder a los proyectos } \\
\text { conjuntos. }\end{array}$ \\
\hline
\end{tabular}

Tabla I. Principales factores que afectaron la construcción de la agenda de innovación

ISSN: 07 I8-2724. (http://www.jotmi.org) 


\subsection{Participación de los actores del sistema de innovación en el establecimiento de la agenda}

Los sistemas de innovación dependen del flujo de información, la tecnología, los recursos, dinero y las personas. Por esta razón, la interacción es la base para la implementación de redes y colaboración que permitan construir capacidades hacia la innovación abierta.

La definición de la agenda y la fase de post-talleres claramente mostraron que la implementación del sistema estatal de innovación requerirá un nuevo patrón de participación. La figura I representa un mapa de la participación de los grupos de interés en el proceso de elaboración de la agenda de innovación.

Se observa que actores importantes como las instituciones financieras participan de manera marginal. Lo mismo puede decirse de las cámaras industriales, instituciones federales relacionadas con políticas industriales y regulaciones $y$ otros agentes de más alto nivel responsables de las políticas públicas.
Las grandes empresas juegan un papel de liderazgo en la promoción de redes sobre todo en los sectores de alta tecnología, actuando como "tractoras", fomentando una cadena de valor integrada por PYMEs y otras instituciones de lyD. Por esta razón, se debería promover un nuevo rol de las empresas grandes, usando, para ello, incentivos adecuados. Lo mismo puede aplicar para las agencias gubernamentales de promoción industrial. El nuevo gobierno del Estado de México comenzó en $201 \mathrm{l}$ y termina en 2017 y la innovación es un componente de la mayoría de las políticas sectoriales. Una mayor participación para la implementación de la agenda de innovación puede proporcionar grandes beneficios a los encargados de las políticas públicas dado que este ejercicio ha generado información útil y un número considerable de actores clave en la industria, pero el principal resultado ha sido una visión compartida de oportunidades y necesidades.

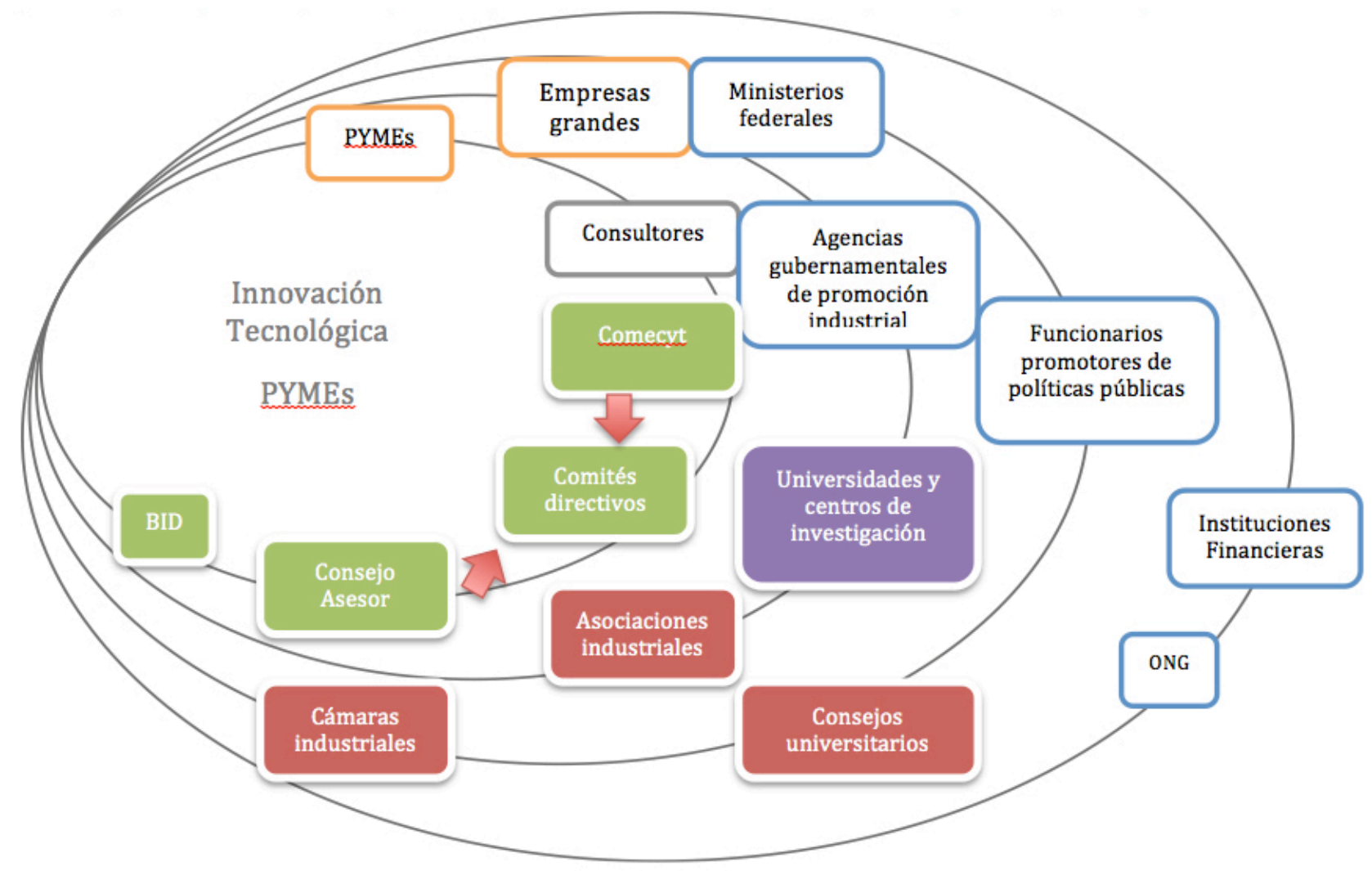

Figura I. Distribución orbital de actores del sistema estatal de innovación en la definición de la agenda

ISSN: 07 I8-2724. (http://www.jotmi.org) 


\subsection{Programa de apoyo para fortalecer la innovación en las PYMEs}

El segundo componente del Programa SIEM se orientó a incrementar la inversión de las PYMEs en actividades de innovación y colaboración para incrementar su competitividad. Se ofrecieron fondos concurrentes para proyectos cooperativos de innovación para promover la inversión privada y los vínculos institucionales. Las empresas podían obtener hasta un $50 \%$ de los costos del proyecto de innovación.

Luego de la definición de la agenda de innovación, el reto fue traducir las grandes áreas de oportunidad en proyectos de colaboración reales, en los cuales al menos se involucraran tres instituciones para contender con el desarrollo tecnológico. El proponente debería ser una PYME del Estado de México de alguno de los sectores prioritarios y los otros dos actores podían ser otras empresas, universidades, centros de investigación, etc.

Para esta nueva etapa se contrataron cinco gestores tecnológicos para apoyar a las empresas en la formulación de los proyectos de acuerdo a las bases de la convocatoria. Esta asistencia fue factor clave para el conformación exitosa de la red de colaboración y generar los propuestas de los proyectos, dado que las PYMEs participantes mostraban un bajo nivel de madurez tecnológica, lo cual se explica en las siguientes subsecciones.

\subsection{Contacto inicial con empresas potencialmente innovadoras}

La primera actividad fue invitar a los directivos de las empresas a un taller donde se explicaban los términos de referencia de la convocatoria y en particular los apoyos económicos ofrecidos por el Programa SIEM. Dado que los directorios públicos de las empresas son poco confiables fue necesario que los consultores generaran sus propias bases de datos mediante un trabajo directo de llamadas. Posteriormente se invitó a las empresas a los talleres mediante correo electrónico pero los resultados fueron pobres, la mayoría de las empresas no respondieron. ${ }^{7}$
Una estrategia para mejorar el acercamiento con las empresas fue hacer sinergia con otros programas de apoyo tecnológico a las PYMEs, concretamente se tuvieron resultados positivos con un programa de la Secretaría de Economía y la Fundación México -Estados Unidos para la Ciencia el cual ya tiene varios años operando en el Estado de México $y$ ha generado un alto nivel de credibilidad entre los empresarios.

La estrategia de los talleres se fortaleció con visitas a las empresas, lo cual dio mayor confianza a los empresarios y mejoró sustancialmente la identificación de proyectos potenciales. Este mecanismo si bien fue de los más útiles tuvo como inconveniente que se requería de mucho tiempo para ejecutarlo.

\subsubsection{Identificación de necesidades tecnológicas}

La identificación de las necesidades tecnológicas alineadas con los objetivos de la agenda de innovación fue una tarea difícil dado que existe una gran confusión sobre el significado del concepto “innovación”. Para la mayoría de las empresas, en el primer acercamiento al Programa SIEM, se contemplaba la oportunidad de acceder a financiamiento para la compra de equipo.

Una vez que se aclaraba la intención del Programa SIEM para apoyar la innovación a través del desarrollo tecnológico, la mayoría de los proyectos que los empresarios ubicaban se asociaban a la innovación de producto, dejando de lado innovaciones de equipo, proceso o incluso organizacionales. En la formulación de los proyectos, fue impactante conocer que la mayoría de los directivos de las empresas desconocían sus mercados y la competencia, por lo que los consultores debieron no sólo llevar a cabo el análisis del estado del arte, sino también una investigación del mercado. Una herramienta que fue utilizada exitosamente en el sector de floricultura fue el mapa de ruta, a través del cual se orientaron los objetivos de mercado y se identificaron los puntos críticos para lograr dichos objetivos.

Una lección importante de esta etapa fue que los gestores de tecnología necesitan una gran flexibilidad. Los objetivos iniciales de los proyectos cambiaron y evolucionaron para cumplir con los criterios establecidos, las fortalezas técnicas de la red y las oportunidades de mercado.

\footnotetext{
${ }^{7}$ Los productores de flores mostraron mucho interés en el Programa; sin embargo, la mayoría de éstos no cumplía con los requerimientos de la convocatoria, pues al no contar con una figura fiscal no eran elegibles. La mayoría de los productores son rurales e informales.
}

ISSN: 07 I8-2724. (http://www.jotmi.org) 


\subsubsection{Identificación de los socios tecnológicos}

A la vez que se establecían los objetivos del proyecto y sus alcances, se buscaron opciones de socios tecnológicos que pudieran ejecutar el proyecto. Se identificaron universidades y centros de investigación que pudieran realizar los trabajos requeridos. Los tiempos de respuesta de estas instituciones fueron muy largos, en la mayoría de los casos y no de acuerdo con las necesidades de las empresas. Respecto a los precios, éstos fueron en general muy elevados, lo que obligó a los consultores a iniciar un proceso de negociación de precios y alcances y buscar otras alternativas de socios. Fue relativamente fácil negociar y llegar a un acuerdo con los proveedores, pero casi imposible lograr acuerdos de colaboración entre empresas del mismo sector, debido fundamentalmente a la falta de confianza y al temor de que se fugara información confidencial.

\subsubsection{Formulación del proyecto}

Esta actividad fue difícil y ocupó mucho tiempo. La mayoría de los directivos de las empresas no tenían la habilidad de traducir su idea en objetivos concretos; así mismo, no estaban familiarizados con los conceptos asociados a la formulación de un proyecto de innovación tecnológica. Otra limitación encontrada fue que en muchos casos el personal de la empresa involucrado en el desarrollo del proyecto, no contaba con suficiente tiempo para esta actividad, esto derivó en que la responsabilidad de la formulación del proyecto recayera en los consultores, quienes tuvieron a su cargo las actividades de concebir el proyecto, distribuir actividades entre los miembros de la red, estimar el presupuesto y negociar los términos de participación de los miembros de la red. En aquellos casos en los que se involucró a universidades, los consultores tuvieron que negociar con las autoridades para cumplir con los requerimientos institucionales y obtener las cartas compromiso.

En tres casos específicos, los directivos de las empresas declinaron continuar con la formulación del proyecto; sin embargo, esta decisión se tomó cuando el proyecto estaba al $95 \%$ de avance. El argumento que se dio fue que los altos directivos no estaban de acuerdo en participar en la convocatoria, este hecho refleja la falta de compromiso con la innovación y de que los empresarios no fueron conscientes del costo asociado a la formulación de los proyectos. ${ }^{8}$

\subsubsection{Formalización de los proyectos: convenios de colaboración}

Como respuesta a la convocatoria de fondos concurrentes, se presentaron 3I proyectos, de los cuales 21 fueron aprobados. Cada proyecto fue revisado por tres evaluadores miembros del Registro Nacional de Evaluadores Acreditados. La comunicación de la aprobación de los proyectos se obtuvo casi al término del año fiscal, por lo que las negociaciones con todos los involucrados tuvo que hacerse de manera simultánea. Uno de los aspectos centrales de las negociaciones fue el relacionado con los derechos de propiedad intelectual. Este punto debió verse caso a caso, sin regla alguna. ${ }^{9}$

\subsection{Lecciones del Programa}

La tabla 2 resume los hallazgos de este análisis. El papel del consultor fue crítico para resolver una serie de dificultades asociadas a la falta de experiencia, credibilidad y compromiso de las PYMEs participantes. En las empresas tecnológicamente maduras, la mayoría de estas actividades fueron responsabilidad del personal de la empresa. Es claro que para que esta iniciativa tuviera éxito fue relevante ofrecer asistencia técnica en todas las fases del proyecto, desde su concepción hasta su ejecución y finalización.

\footnotetext{
${ }^{8}$ El costo de la asesoría técnica de los consultores fue pagada al $100 \%$ con los recursos aportados por las entidades participantes (BID-COMECYT-CONACYT) y los empresarios no tuvieron que erogar nada por este concepto.
}

\footnotetext{
9 En este momento los proyectos aprobados están ejecutándose y se ha pasado a la siguiente etapa del Programa que es el seguimiento de actividades y evaluaciones técnico - financieras. La mayor parte de los proyectos concluirán en 2014.
} 


\begin{tabular}{|c|c|c|c|}
\hline Etapa del proyecto & Fortalezas & Limitaciones & Función del gestor tecnológico \\
\hline Contacto inicial & $\begin{array}{l}\text { Trabajo previo para iden- } \\
\text { tificación de la agenda de } \\
\text { innovación } \\
\text { Posibilidades de financia- } \\
\text { miento }\end{array}$ & $\begin{array}{l}\text { Información sobre las } \\
\text { empresas poco confiable } \\
\text { Poca confianza en las } \\
\text { instituciones guberna- } \\
\text { mentales }\end{array}$ & $\begin{array}{l}\text { Creación de sinergias con } \\
\text { otros programas } \\
\text { Elaboración de bases de datos } \\
\text { confiables }\end{array}$ \\
\hline $\begin{array}{l}\text { Identificación de necesi- } \\
\text { dades tecnológicas }\end{array}$ & $\begin{array}{l}\text { Interés de los directi- } \\
\text { vos para obtener apoyo } \\
\text { financiero }\end{array}$ & $\begin{array}{l}\text { Poco entendimiento de } \\
\text { los conceptos básicos de } \\
\text { innovación } \\
\text { Confusión de necesi- } \\
\text { dades críticas } \\
\text { Poco conocimiento so- } \\
\text { bre tendencias técnicas y } \\
\text { de mercado }\end{array}$ & $\begin{array}{l}\text { Análisis del estado del arte } \\
\text { Evaluación del mercado poten- } \\
\text { cial } \\
\text { Colaboración con personal de } \\
\text { la empresa para hacer explíci- } \\
\text { tos las necesidades técnicas y } \\
\text { los objetivos del proyecto. } \\
\text { Elaboración de mapas de ruta }\end{array}$ \\
\hline $\begin{array}{l}\text { Identificación de socios } \\
\text { potenciales }\end{array}$ & $\begin{array}{l}\text { Participación de empre- } \\
\text { sas e instituciones en la } \\
\text { definición de la agenda } \\
\text { Colaboración con prov- } \\
\text { eedores para asistencia } \\
\text { técnica } \\
\text { Contactos con investi- } \\
\text { gadores universitarios }\end{array}$ & $\begin{array}{l}\text { Respuesta lenta de } \\
\text { universidades y otras } \\
\text { instituciones } \\
\text { Rechazo de las empresas } \\
\text { a colaborar con otras } \\
\text { empresas }\end{array}$ & $\begin{array}{l}\text { Elaboración de mapas sobre } \\
\text { posibles socios, basados en el } \\
\text { mapa de ruta. } \\
\text { Negociación de colaboraciones }\end{array}$ \\
\hline Formulación del proyecto & $\begin{array}{l}\text { Interés de los partici- } \\
\text { pantes }\end{array}$ & $\begin{array}{l}\text { Poca experiencia de los } \\
\text { directivos de empresas } \\
\text { para escribir propuestas } \\
\text { Poco tiempo dedicado a } \\
\text { escribir la propuesta } \\
\text { Datos poco confiables } \\
\text { sobre el mercado, costos } \\
\text { internos y flujo de efec- } \\
\text { tivo }\end{array}$ & $\begin{array}{l}\text { Identificación de objetivos, } \\
\text { roles, presupuestos y agenda. } \\
\text { Borrador de la propuesta } \\
\text { Creación de consenso alrede- } \\
\text { dor de la propuesta }\end{array}$ \\
\hline $\begin{array}{l}\text { Formalización de acuer- } \\
\text { dos }\end{array}$ & $\begin{array}{l}\text { Los proyectos aproba- } \\
\text { dos tenían asegurado la } \\
\text { provisión de recursos }\end{array}$ & $\begin{array}{l}\text { Poco tiempo } \\
\text { Negociaciones bajo } \\
\text { estrés }\end{array}$ & $\begin{array}{l}\text { Borradores de los acuerdos } \\
\text { Negociación } \\
\text { Distribución "justa" de los } \\
\text { derechos de propiedad intelec- } \\
\text { tual }\end{array}$ \\
\hline
\end{tabular}

Tabla 2. Fortalezas, limitaciones y la función del gestor tecnológico. 


\section{Conclusiones}

El programa SIEM ha sido una iniciativa importante para promover la innovación y la colaboración entre los distintos actores del sistema de innovación. El Programa con un presupuesto bajo (aproximadamente dos millones de dólares) ha motivado la inversión privada para desarrollo tecnológico en aproximadamente 4 millones.

Las PYMEs participantes han podido constatar que los objetivos de innovación tecnológica pueden derivar en un incremento de competitividad y nuevas oportunidades de negocio. Sin embargo, los recursos para proyectos cooperativos de innovación ofrecidos a las PYMEs como un incentivo económico son insuficientes, particularmente para los sectores químico y farmacéutico donde los proyectos requieren de presupuestos mayores. Deben existir mecanismos de apoyo diferenciados.

La generación de capacidades ha sido identificado como un factor de éxito del Programa SIEM y por ello la capacitación de alto nivel en temas relacionados con políticas de innovación se han implementado para personal de las empresas, universidades, centros de lyD e instituciones gubernamentales. Para obtener beneficios tangibles debe asegurarse la continuidad de esta capacitación.

Se requiere involucrar a los tomadores de decisiones de alto nivel, en materia de políticas públicas, para plasmar la agenda en proyectos factibles. Por ello, debe mejorarse la estrategia de comunicación. Se requieren mayores recursos para las innovaciones y los actores del sistema deben utilizar estrategias más proactivas para convencer a las autoridades de la importancia de un mayor compromiso con la innovación y la competitividad de las PYMEs.

La burocracia tiene impactos negativos que repercuten en retrasos e ineficiencias. Factores positivos están asociados con incentivos económicos directos y asistencia técnica para concebir e implementar los proyectos. El marco de políticas públicas proporciona una orientación estratégica; sin embargo, la generación de consensos es una tarea difícil en los sectores dada la heterogeneidad de éstos y la falta de una cultura de colaboración. 


\section{Referencias}

ASHEIM, B.; ISAKSEN, A.; NAUWELAERS, C.; TÖDTLING, F. (2003): Regional Innovation Policy for Small-Medium Enterprises. Edward Elgar Publishing, Inc. Massachusetts, U.S.A.

CHECKLAND, P. (1999). Systems Thinking, Systems Practice: Includes a 30-Year Retrospective, John Wiley \& Sons Ltd.

COOKE, P. (2002). Knowledge Economies. Clusters, learning and cooperative advantage, Routledge, London.

CORDIS (2004). Technology Platforms : from Definition to Implementation of a Common Research Agenda, European Commission, EUR 21265, Brussels. Consultado el 14 de marzo de 2013 en <ftp://ftp.cordis.europa.eu/pub/ technology-platforms/docs/tp_report_defweb_en.pdf>.

DINI, M.; FERRARO, C.; and GASALY, C. (2007). Pymes y articulación productiva. Resultados y lecciones a partir de experiencias en América Latina. Desarrollo productivo series, 180, Economic Commission for Latin America and the Caribbean (ECLAC), United Nations publication, Santiago, Chile.

Economic Commission for Latin America and the Caribbean (ECLAC), Inter-American Development Bank (IDB) and Organization of American States (OAS). (20I I). Innovating, Gaining Market Share and Fostering Social Inclusion Success Stories in SME Development, ECLAC/UN/IDB/OAS, Santiago, Chile. Consultado el 2 de abril de 2013 en <http:// www/iadb.org/document.cfm?id=36599452>.

Economic Commission for Latin America and the Caribbean (ECLAC). (2010). Time for equality: closing gaps, opening trails. Santiago, Chile.

HAE, S. J. (2006). Regional Innovation System and Industrial Cluster: Its Concept, Policy Issues and Implementation Strategies in NationalWorkshop on Sub-national Innovation Systems and Technology Capacity Building Policies to Enhance Competitiveness of SMEs, Beijing, China, 27-30 October.

Instituto Mexicano para la Competitividad (2010). Índice de Competitividad Estatal 2010. La Caja Negra del Gasto Público. México. Consultado el 7 de marzo de 2013 en $<$ http://imco.org.mx/indice_estatal_2010>.

ISAKSEN,A. (200I). Building Regional Innovation Systems: Is Endogenous Industrial Development Possible in the Global Economy. Canadian Journal of Regional Science, I, I0I-I20.
LAGENDIJK, A. (2000). Learning in Non-core Regions: Towards ,Intelligent Clusters'; Addressing Business and Regional Needs, in F. Boekema, K. Morgan, S. Bakkers and R. Rutten (Eds.), Knowledge, Innovation and Economic Growth, Edward Elgar Publishing. London, U.K. 165-191.

LEWIN, K. (1946). Action Research and Minority Problems. Journal of Social Issues. Vol. 2, Issue 4, 34-46.

LEWIN, K. (1958). Group Decision and Social Change. Holt, Rinehart and Winston. New York.

LUNDVALL, B.-A. (1992) National Systems of Innovation: Towards a Theory of Innovation and Interactive Learning, Pinter, London.

OECD (2009). OECD Reviews of Regional Innovation: 15 Mexican States 2009. Consultado el 22 de febrero de 2013 en <http://www.oecd.library.org/docserver/ download/040906 le.pdf>

SOLLEIRO, J.L.; GAONA, C. (20I2). Promotion of a regional innovation system: the case of the State of Mexico. Social and Behavioral Sciences 52, $110-119$.

TÖDTLING, F.; TRIPPL, M. (2005). One size fits all? Towards a differentiated policy approach with respect to regional innovation systems. Research Policy, 34 (8), I203-1219. 